\title{
Kinetics of SARS-CoV-2-Neutralising Antibodies of Residents of Long-Term Care Facilities
}

\author{
J. Moyet ${ }^{1}$, F. Helle ${ }^{2}$, G. Bourdenet ${ }^{3,4}$, C. Joseph ${ }^{2,5}$, B. Gubler ${ }^{3,4,6}$, G. Deschasse ${ }^{1}$, I. Defouilloy ${ }^{1}$, T. Slovenski ${ }^{1}$,

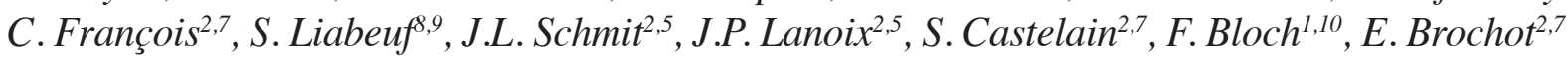

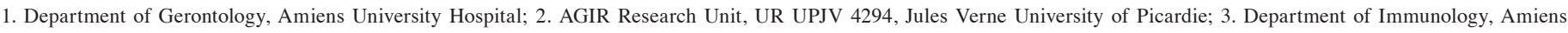

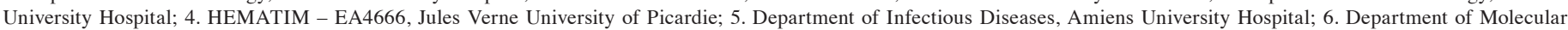

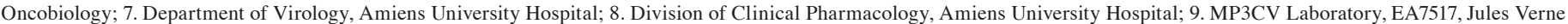
University of Picardie; 10. Laboratory of Functional Neurosciences UR 4559, Jules Verne University of Picardie, Amiens, France.

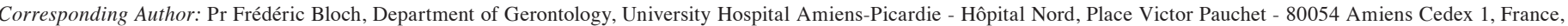
Phone / Fax: +333.22.45.57.20 / +333.22.45.53.30, E-mail: bloch.frederic@chu-amiens.fr, ORCID identifier : 0000-0002-6046-7097

\begin{abstract}
INTRODUCTION: Elderly residents of nursing homes (NHs) and long-term care units (LTCUs) have been shown to have a high risk of mortality and morbidity in cases of SARS-CoV-2 infection. The objective of this study was to examine the kinetics of neutralizing antibodies (NAbs) directed against the SARS-CoV-2 virus in residents of the NH and LTCU units of our University Hospital who were identified with positive serology after the first epidemic outbreak.

MATERIALS AND METHODS: The participants included were sampled every three months for qualitative serological testing, as well as quantitative testing by neutralization tests using retroviral particles containing the S glycoprotein of SARS-CoV-2. Vaccination using the Comirnaty (Pfizer BNT162b2) vaccine begun before the last serological follow-up.

RESULTS: The median NAb titer in June 2020 was 80 [40; 60] versus $40[40 ; 160]$ three months later, showing a statistically significant decline $(\mathrm{p}<0.007)$, but remained stable between the three- and sixmonth timepoints $(\mathrm{p}=0.867)$. By nine months after vaccination, we observed a significant difference between vaccinated residents known to have positive serology before vaccination (SERO+, Vacc+) and those vaccinated without having previously shown COVID-19 seroconversion (SERO-, Vacc+), the latter group showing similar titers to the SERO+, Vacc- participants $(\mathrm{p}=0.166)$. The median antibody titer in SERO+, Vacc+ patients increased 15 -fold following vaccination.

DISCUSSION: Humoral immunity against SARS-CoV-2 appears to be persistent in elderly institutionalized patients, with a good post-vaccination response by residents who had already shown seroconversion but a notably diminished response by those who were seronegative before vaccination. To evaluate immunity in its entirety and elaborate a sound vaccination strategy, the cellular immune response via $\mathrm{T}$ cells specific to SARS-CoV-2 merits analysis, as this response is susceptible to being affected by immunosenescence.
\end{abstract}

Key words: SARS-CoV-2, COVID-19, nursing homes, immunosenescence, serological assay.

\section{Introduction}

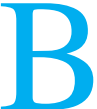
eginning December 2019, an emerging viral disease tied to the newly discovered SARS-CoV-2 coronavirus was identified and has henceforth caused a global pandemic, with important health-related, economic, demographic, and social consequences $(1,2)$. The aforementioned viral infection (COVID-19), characterized by respiratory tropism via binding of the Spike protein of SARS-CoV-2 by the ACE2 receptor and high transmissibility, is currently responsible for more than four million deaths worldwide, with mortality increasing with age $(2,3)$. The elderly and the frail have been the most affected by the severity and lethality of this disease (4). The residents of nursing homes (NHs) and long-term care units (LTCUs) have a high risk of mortality in case of an infectious cluster in their respective institutions because of their medical profile (5). Furthermore, diverse clinical presentations of the disease have been observed in the elderly, making the diagnosis difficult and potentially causing delays in medical treatment and precautionary isolation in the abovementioned establishments.

Given the magnitude and length of this pandemic, many clinical trials and observational studies have enabled better management of the disease, notably through its prevention by means of efficient vaccination using vaccines obtained by new technologies involving mRNA (6). The persistence and long-term efficiency of immunity acquired by infection or vaccination are not yet fully understood. It appears that the perspective of herd immunity, capable of limiting the epidemic in the face of newly emerging variants, is dependent on the persistence of protective or neutralizing antibodies NAbs in people infected by, or vaccinated against, SARSCoV-2 $(7,8)$. Multiple studies have proven the effectiveness of these new vaccines in preventing reinfection by SARS-CoV-2 when administered in two doses and, moreover, that a single dose should suffice for patients who have been infected and cured (9-11). Nevertheless, there is currently little data on the intensity and efficiency of the immune response to COVID-19 infection or vaccination in elderly, frail patients. The steady increase in life expectancy over the last century is, without a doubt, the expression of longer and healthier lives. It should, however, prompt an analysis through the lens of physiological ageing and, more explicitly, immune ageing. The dysregulation of the immune system with ageing, immunosenescence, is a process that contributes to the increase in the incidence of infectious disease and a diminished vaccine response, while also playing a role in the development of auto-immune diseases, neoplasia, and neurodegenerative diseases (12-15). 
The NHs and LTCUs of our University Hospital have faced an exceptional number of suspected or confirmed cases during this period. This was confirmed by a screening campaign that took place in June 2020 and which found 147 of 221 residents $(66.5 \%)$ with positive serological markers for prior SARS$\mathrm{CoV}-2$ infection. The purpose of the SERO-CoV-OLD study was to determine the presence and quantitative evolution of neutralizing COVID-19 antibodies (NAbs) among residents living in the NHs and LTCUs of our University Hospital.

\section{Materials and methods}

\section{Study design}

This was a descriptive prospective cohort study classified as Category 2 according to French law on human research. In June 2020, our University Hospital organized a COVID-19 screening campaign in the heart of its LTCUs $(n=5)$ and NHs $(n=5)$ aiming to evaluate the impact of the first epidemic outbreak in these facilities. This campaign was based on the use of RT-PCR tests (Abbott Alinity SARS-Cov-2 IgG assays) and ELISA (Wantai SARS-CoV-2 Ab ELISA). The screening took place on June 15 to 16, 2020, enrolling 221 patients. Faced with a high seropositivity rate $(66.5 \%)$, we decided to follow-up the patients by repeated serological determinations every three months for a period of nine months, while also using the samples to quantify NAb levels. The inclusion criteria consisted of residing in one of the hospital NHs or LTCUs and having participated in the June 2020 screening campaign. The SERO-CoV-OLD study protocol was approved by the ethics board of our University Hospital (PI2020-843_0079) (ID ClinicalTrials.gov: NCT04563650) on July 15, 2020. Of note, during the development and validation of the protocol, vaccination against COVID-19 was not yet available, and thus, it was not considered. The study volunteers were vaccinated between the beginning of January and the middle of February 2021 with the Comirnaty vaccine (Pfizer BNT162b2), the second dose administered 21 days later.

\section{Clinical information}

The participants' clinical and epidemiological data were extracted from the electronic medical record, with special attention paid to a prior medical history concerning cardiovascular, respiratory, oncological, and autoimmune disease, shown to be linked to an increased risk of morbidity and mortality in the context of SARS-CoV-2 infection. The nutritional, cognitive, and autonomy profiles were also assessed using the Rockwood scale (16). We also screened the medical records for symptoms suggestive of COVID-19 infection, as well as those of contacts of confirmed patients or RT-PCR tests given during the first outbreak.

\section{Serological tests and sampling schedule}

Patients included in the protocol and concomitantly shown to have positive serology in June 2020 were chosen to be tested using qualitative serological tests to detect antibodies against the SARS-CoV-2 nucleocapsid (Abbott Alinity SARSCov-2 IgG assay) and Spike proteins (Wantai SARS-CoV-2 Ab ELISA) every three months, according to the following scheme: in June 2020 (M0), and three (M3), six (M6) and nine months later (M9). Only the serologically positive participants participated in the M3 and M6 samplings. All patients included in the protocol who received two Comirnaty (Pfizer BNT162b2) vaccine doses were also serologically screened to determine the impact of vaccination on the immune response.

\section{Neutralization tests}

Serum neutralization assays, using a non-infectious pseudo virus, were carried out to determine the presence of NAbs and their titer. Pseudotyped retroviral particles containing SARSCoV-2 virus $\mathrm{S}$ glycoprotein (SARS-CoV-2pp) were produced, as described before $(17,18)$, by transfection with a plasmid encoding the $\mathrm{S}$ glycoprotein of SARS-CoV-2 and a reporter plasmid encoding the luciferase enzyme. The pseudotyped particles were collected 48,72, and $96 \mathrm{~h}$ after filtration through a $0.45-\mu \mathrm{m}$ pore filter. The plasma obtained for the serological tests was diluted by two-fold serial dilutions and then incubated with the SARS-CoV-2pp for $1 \mathrm{~h}$ before being put in contact with 293T( ATCC® CRL-3216TM) cells that had been transitorily transfected $24 \mathrm{~h}$ before with a plasmid encoding the human ACE2 receptor protein (pcDNA3.1-hACE2). The luciferase activity was measured after $72 \mathrm{~h}$ of incubation. The NAb titer was defined as the plasma dilution that inhibits $50 \%$ of the luciferase activity. The tests were independently performed twice. The specificity of the neutralization was tested beforehand on plasma infected by other coronaviruses and pseudotyped retroviral particles of the vesicular stomatitis virus (VSV). Every sample was tested twice, in the same series and in two different series, to determine a mean titer value.

\section{Data collection and statistical analysis}

For the description of the clinical and biological characteristics of the participants, quantitative variables are described as means \pm standard deviations or medians (interquartile ranges) and were compared using Student t, Mann-Whitney, or Kruskal-Wallis tests. The qualitative variables are described by their number and frequency and were compared between the two groups using the $\chi^{2}$ or Fischer's exact test.

\section{Results}

\section{Clinical characteristics of included residents}

During the inclusion period, 97 residents living in the 10 units were included, representing $44 \%$ of the 221 residents who participated in the June screening campaign. Their clinical and epidemiological characteristics are presented in Table 1. The division between the host facilities was as follows: $58(60 \%)$ hosted in LTCUs and 39 in NHs. The mean age was 87.3 ( \pm 
6.6) years in the LTCUs and $87.6( \pm 6.7)$ in the NHs $(\mathrm{p}=0.7)$. Most participants (77) were women, accounting for $77 \%$ of the population. The male/female distribution was similar for the two types of units $(\mathrm{p}=0.159)$. The residents were very frail, with a median Rockwood score of 7 [7; 8] indicating severe frailty. The LTCU residents were determined to be frailer than the NH residents based on the Rockwood scale $(\mathrm{p}<0.001)$. From a nutritional point of view, the mean bodyweight was 64.1 $( \pm 19.6) \mathrm{kg}$ for an albumin level of $32.7( \pm 5.2) \mathrm{g} / \mathrm{l}$. In terms of medical history, there was a high prevalence of neurocognitive disease $(n=83[86 \%])$, followed by hypertension $(n=63$ $[65 \%])$ and a history of cardiovascular events $(n=33[34 \%])$, predominating in the LTCU $(\mathrm{p}=0.001)$. The overall burden of COVID-19 seroprevalence was $64 \%$, with a higher positivity rate in the NHs $(92 \%)$ than LTCUs $(45 \%)(\mathrm{p}<0.001)$.

\section{Figure 1. Evolution of COVID-19 seroprevalence in the NHs and LTCUs}

\begin{tabular}{|c|c|c|c|c|}
\hline \multicolumn{5}{|c|}{ Evolution of COVID 19 seroprevalence } \\
\hline \multicolumn{5}{|l|}{80} \\
\hline \multicolumn{5}{|l|}{70} \\
\hline \multicolumn{5}{|l|}{60} \\
\hline \multicolumn{5}{|l|}{50} \\
\hline $\begin{array}{l}40 \\
30\end{array}$ & \multicolumn{4}{|c|}{30} \\
\hline \multicolumn{5}{|l|}{20} \\
\hline \multicolumn{5}{|l|}{10} \\
\hline \multirow[t]{2}{*}{0} & & & & \\
\hline & $\begin{array}{c}\begin{array}{c}\text { ELISA June } \\
n=97\end{array}\end{array}$ & ELISA M3 $n=58$ & ELISA M6 $n=45$ & $\begin{array}{c}\text { ELISA M9 post } \\
\text { vaccination } \\
n=81\end{array}$ \\
\hline NEGATIVE & 35 & 12 & 0 & 6 \\
\hline I POSITIVE & 62 & 46 & 45 & 75 \\
\hline
\end{tabular}

\section{Evolution of the prevalence of COVID-19 seropositivity (Figure 1)}

Among the 97 patients included in June 2020, 62 (64\%) showed positive COVID-19 serology. At the M3 time point, 58 of the $62(94 \%)$ patients who were positive in June were sampled again and $46(79 \%)$ had serological tests showing the presence of SARS-CoV-2 antibodies. The test was negative for the other $12(21 \%)$. At the M6 time point, 45 of the 46 residents who were positive at M3 were able to be retested and all (100\%) tested positive. After the vaccination campaign that took place between January and February 2021 with the Comirnaty (Pfizer BNT162b2) vaccine, 69 (85\%) of the 81 residents sampled at the M9 timepoint had received two vaccine doses. Among them, 75 (93\%) serological tests showed a positive result, 67 for residents having completed the vaccination and eight for non-vaccinated residents who already had positive serological tests at the M3 and M6 samplings. Of note, among the six seronegative residents at M9, two had received two Comirnaty (Pfizer BNT162b2) doses and four were also seronegative in June 2020 and did not receive the vaccine.
Figure 2. Neutralising antibody (NAb) titers in positive patients at the moment of the June screening campaign and their evolution

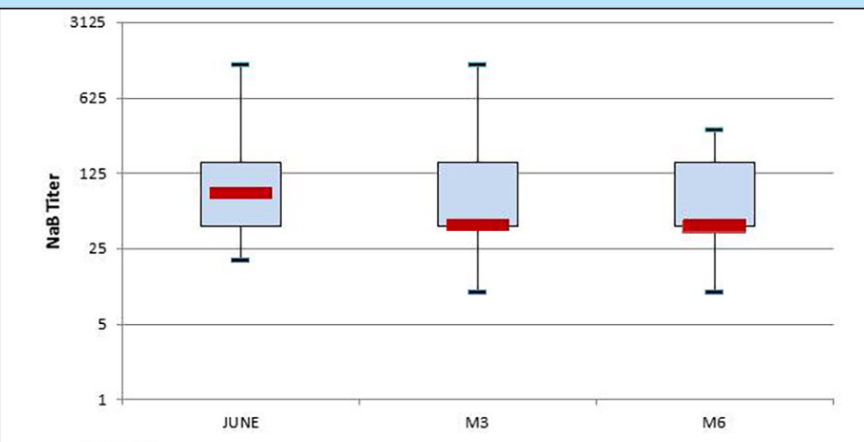

Figure $2 \mathrm{~A}$
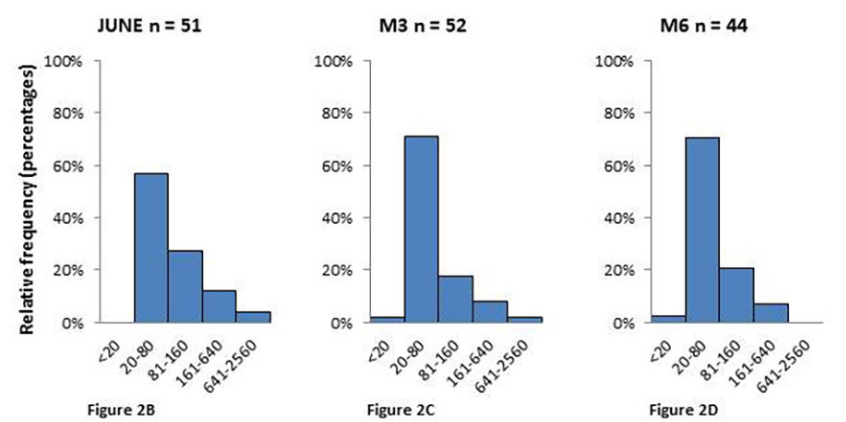

(A) Box plot showing the evolution of median NAb titers over six months, with medians and IQRs and minimal and maximal values at the extremities. (B-D) Distribution of titers (\%) in June (B), at M3 (C), and at M6 (D)

\section{SARS-CoV-2 antibody titers and their evolution (Figure 2)}

We were able to determine the median NAb titer in June for 51 patients, which was found to be 80 [40; 160] (Figure 2A), with 29 (57\%) showing low titers with values between 20 and 80 (Figure 2B). The NAb titer for eight (16\%) of the residents was $>160$, with a maximum titer of 1,280 for two of the participants. At M3, the median NAb titer of the 52 processed samples was calculated to be 40 [40; 160], which was significantly lower than at M0 (p < 0.007) (Figure 2A). Most patients $(38[73 \%])$ showed a NAb titer $<80$ (Figure 2C). Only five $(10 \%)$ residents had titers $>160$, with a maximum of 1,280 for one (Figure 2B). By contrast, the neutralizing antibody titer was stable between M3 and M6 ( $\mathrm{p}=0.867)$, with a median titer of $40[40 ; 160]$. At the M6 timepoint, 41 (92\%) residents had a titer $<160$ and $32(72 \%)<80$, with the lowest titer found being 10 . The maximal titer, found for three residents, was 320 (Figure 2D).

\section{Neutralizing antibody titers and the impact of vaccination (Figures 3 and 4)}

Vaccination with the Comirnaty (Pfizer BNT162b2) vaccine was carried out between mid-January to mid-February, 2021, for 70 included residents $(72 \%)$, with a median time interval before the second dose of 21 days $[21 ; 21]$. The median time interval between the second vaccine dose and the serological 
Table 1. Characteristics of included patients and their repartition between the two participating units

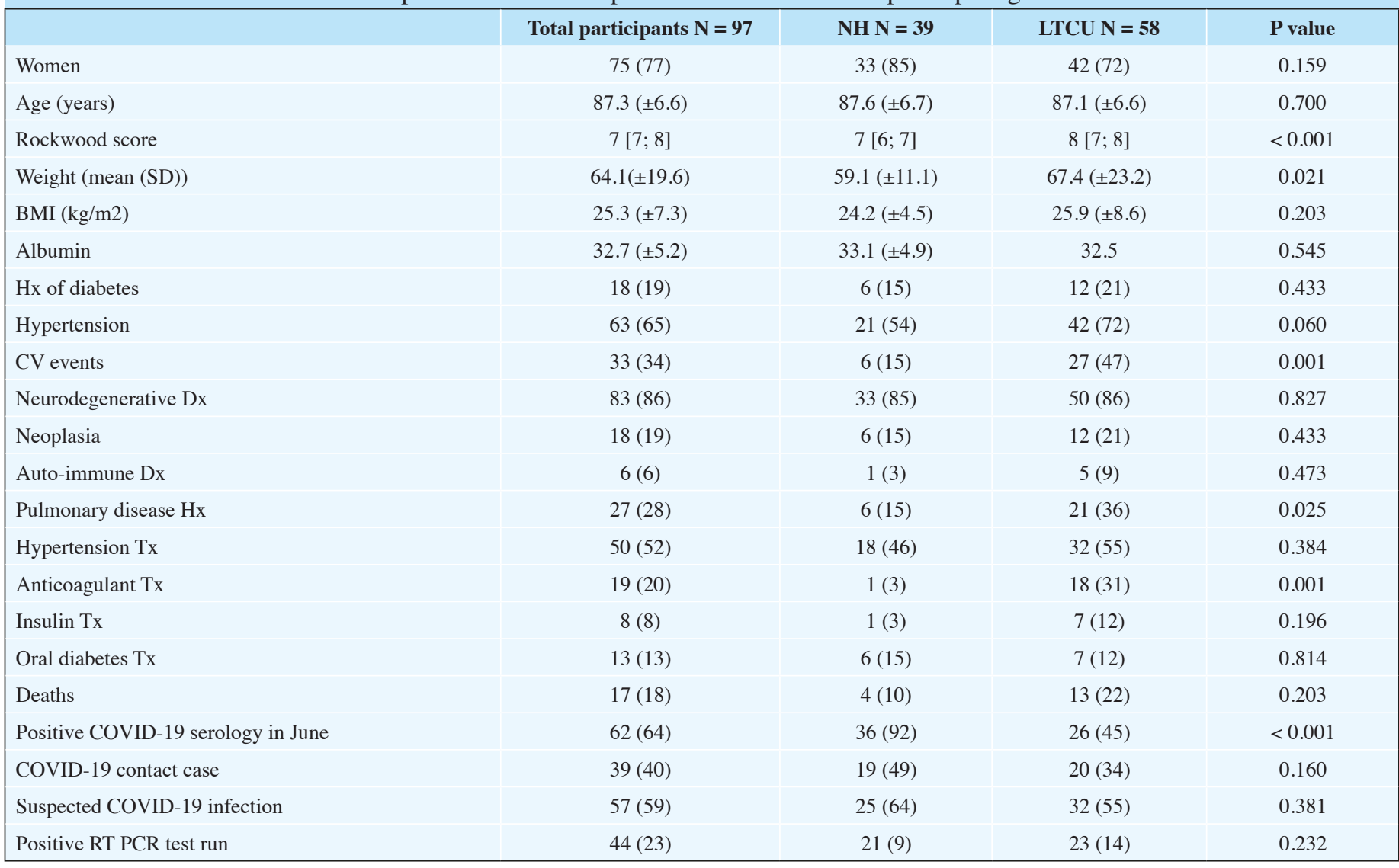

The data are presented as medians (IQRs) or frequencies (percentages)

sampling at M9 was 55 days [50; 60]. NAb titer determination was possible for 71 participants in the study, of whom 44 (62\%) had already shown positive COVID-19 serology in June and received both vaccine doses (SERO+, Vacc+), 19 (27\%) negative serology in June and received both vaccine doses (SERO-, Vacc+), and finally eight (11\%) positive serology in June but did not receive the vaccine (SERO+, Vacc-).

The median neutralizing antibody titer of the SERO+, Vacc+ group was 640 [320; 800], with $37(84 \%)$ patients showing a titer $>160$ and $11(25 \%)$ a titer even higher than 640, with a maximum of 2,560 (figure 3A, 3C). In the SERO-, Vacc+ group, the median titer of 20 [20-40] was significantly lower than that for the SERO+, Vacc+ residents $(\mathrm{p}<0.001)$, with a distribution showing titers of mostly $<80$ (figure 3A, 3D). Two of the SERO-, Vacc+ participants had a very low NAb titer $(<$ 20), having already shown negative M9 serology. The SERO+, Vacc- participants had a median titer of $40[40 ; 50]$, with no statistically significant difference from the SERO-, Vacc+ group $(\mathrm{p}=0.166)$ and with a $100 \%$ distribution between titers of 20 and 80 (figure $3 \mathrm{~A}, 3 \mathrm{~A}$ ). There was a significant difference between the median titers of the SERO+, Vacc+ and SERO+, Vacc-groups $(\mathrm{p}<0.001)$.

We equally quantified the humoral response via the increase in NAb titer at M6 and M9 for residents who had a positive titer at M6 and who had since received the vaccine $(\mathrm{n}=37)$. The median titer was $60[35 ; 160]$ and rose to $640[320 ; 1280]$, for an increase of 15 fold [ 3 fold; 15 fold] (Figure 4).

Analysis of the vaccine response in the SERO+, Vacc+ group showed no difference between the median NAb titers, neither according to the sex of the residents $(p=0.400)$ nor the type of unit that hosted them. However, there was a significant difference in median NAb titers according to age $(p=0.009)$.

\section{Discussion}

\section{The kinetics of neutralizing SARS-CoV-2 antibodies}

Serological monitoring for the presence of anti-SARS-CoV-2 NAbs in the frail and elderly residing in care facilities showed their persistence during a nine-month follow-up period. The June 2020 screening campaign evaluated the first epidemic outbreak, which affected our region between March and April 2020, thus allowing us to highlight the persistence of these antibodies over a period of at least a year, although pertinent serological follow-up could not be carried out for the entire period on more than eight residents due to the rest being vaccinated before M9. Similar timeframes have been described in the relevant scientific literature, with study follow-up ranging from 8 to 13 months (19-21). However, up until now, very few have focused on the elderly, who present with frailty, a high disease burden, and dependency. The NAb titer alone does not demonstrate effective immunity and, conversely, a negative test does not mean that immunity is absent, as it does not account for all components of adaptive immunity. Despite their persistence over time, we cannot affirm that this level of 
neutralizing antibodies confers full protection against COVID19 reinfection, as the memory B-cell humoral response may, by itself, be insufficient, as often described in the context of other viral infections (22).

Figure 3. Neutralising antibody $(\mathrm{Nab})$ titers and their repartition after vaccination

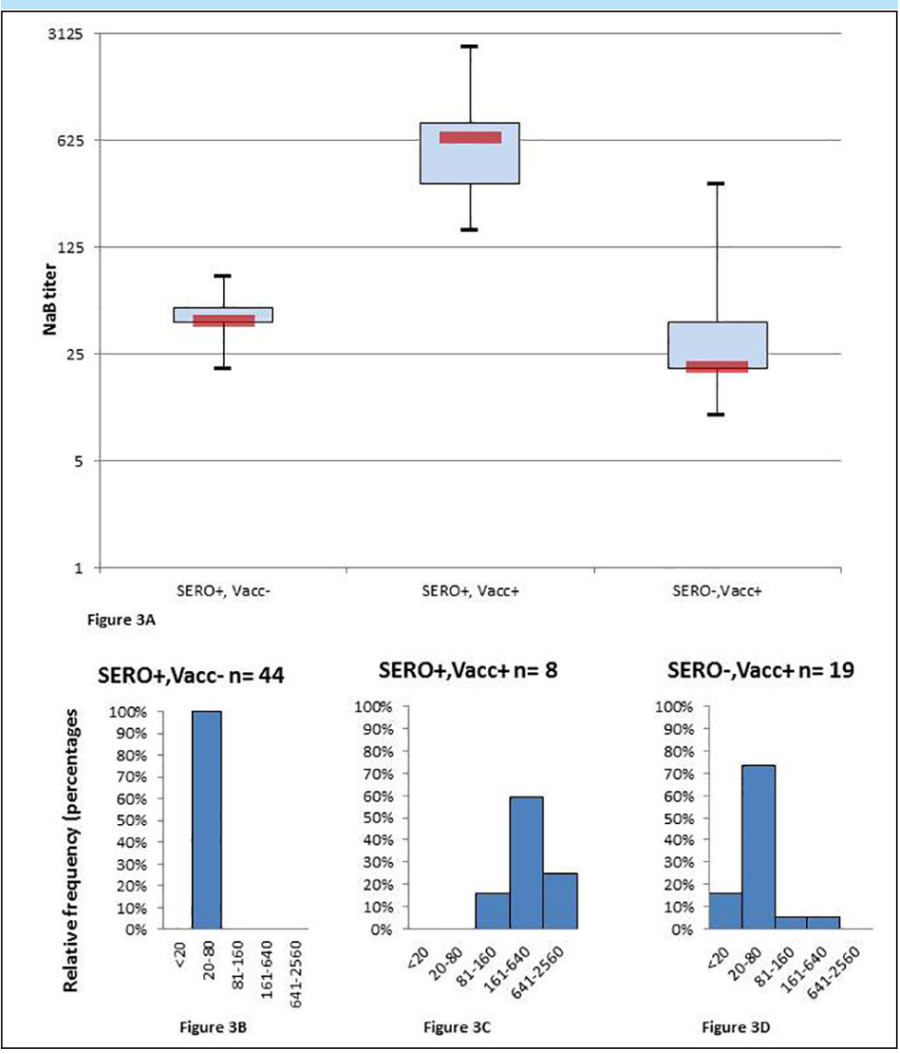

(A) Box plot with the median NAb titers and IQRs and minimal and maximal values at the extremities for the SERO+, Vacc-; SERO+, Vacc+; and SERO-, Vacc+ groups. (B-D) Distribution, in percentages, of NAb titers in the (B) SERO+, Vacc-; (C) SERO+, Vacc+; and (D) SERO-, Vacc+ groups.

It is worth mentioning that the absence of anti-SARSCoV-2 IgG antibodies after pauci-symptomatic infection has been observed in many studies (23). In our study, all residents developed NAbs, as they had symptoms of variable intensity when infected with this pathogen, which is capable of causing diverse symptoms or, sometimes, none at all. During the first SARS-CoV 2 infection outbreak, which was very prevalent in our establishment $(66.5 \%)$, the patients all appear to have developed a humoral response, regardless of the experienced symptoms. We furthermore analyzed the NAb titer of the 147 residents who tested positive in the June campaign and found a significant difference between the median titers of NHs and LTCUs $(p=0.040)$. It is possible that this difference in the humoral response, was due to higher viral circulation in the NHs, with a prevalence of $88.7 \%$ versus $45.6 \%$ in the LTCUs $(\mathrm{p}<0.001)$. Moreover, the LTCU residents in our study showed higher dependency, evaluated by the Rockwood scale ( $\mathrm{p}<$ 0.001 ), potentially linked to more advanced immunosenescence.

Indeed, there was a tight correlation between the qualitative serological tests for the detection of anti-SARS-CoV-2 nucleocapsid antibodies (Abbott Alinity SARS-Cov-2 IgG assay) and anti-Spike protein (Wantai SARS-CoV-2 Ab ELISA) and the neutralization tests, with no false positives encountered. If harmonized and analyzed quantitatively, these tests would allow the monitoring of seroconversion, which is difficult using neutralization tests, which are known to be laborious and hard to implement in most laboratories.

Figure 4. Evolution of neutralizing antibody (Nab) titers (medians and IQs and minimal and maximal values at the extremities) between M6 and M9 for residents who received two Comirnaty (Pfizer BNT162b2) vaccine doses

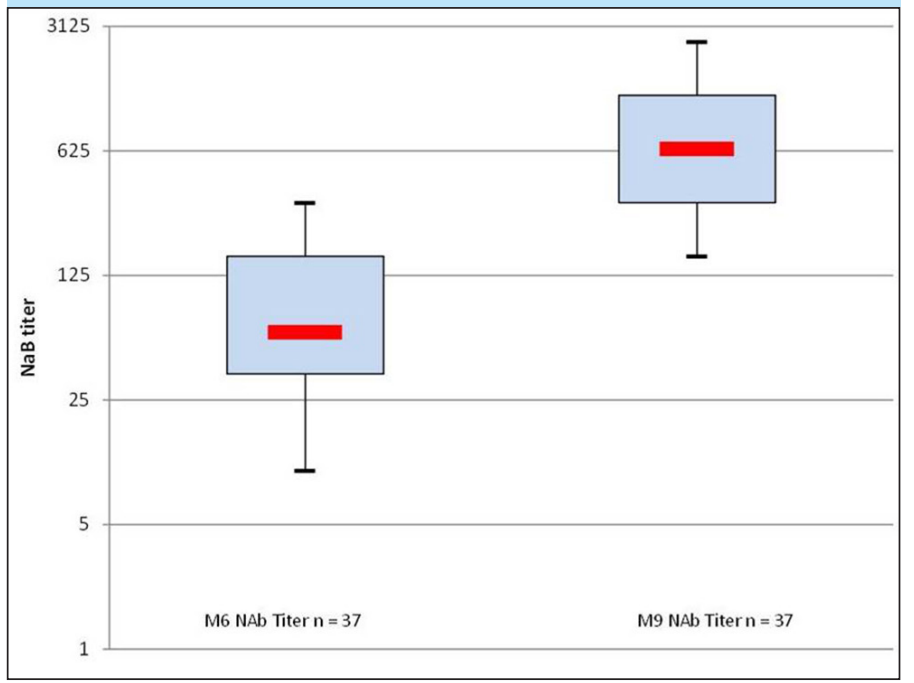

\section{The post-vaccination immune response and neutralizing antibodies}

Analysis of the humoral response by comparing the $\mathrm{NAb}$ titer two months after vaccination with those originally obtained in seropositive patients a few weeks before the first dose showed a 15 -fold [3-fold; 15 -fold] increase in the titer following vaccination. This high post-vaccination titer found in our study may indicate longer lasting and more effective immunity. The possibility of a single-dose vaccination regimen in such COVID-19 seropositive patients could thus be considered, as in the general population, considered to be immunocompetent $(10,11)$. However, there is currently little data on elderly patients, and even less on aged, immunosenescent, and frail populations is available. Furthermore, our analysis of this phenomenon was carried out on a small patient sample using a single vaccine formulation. In contrast to the initially seropositive population, there was a much lower immune response in the seronegative population, with titers comparable to those of non-vaccinated residents who underwent COVID-19 seroconversion. A vaccination schedule containing supplementary doses could potentially be proposed, as already for immunodeficient or immunosuppressed patients younger than 55 .

In a second phase of the SERO-CoV-OLD study, we evaluated the lymphocyte profile of the study participants to analyze the impact of the potential immunosenescent state on the immune response. We explored cellular and humoral immunity independently of SARS-CoV-2 serological status by lymphocyte flow cytometry, as selected circulating 
cytokines are known to have a role in the immune response and immunosenescence. Although the results of this analysis are still being examined, and will be the subject of a separate paper, there appeared to be a decrease in the proportion of naïve $\mathrm{T}$ cells, especially CD8+ T cells. This decrease appears to correlate with a profile of chronic inflammation. Such an association has been previously described in immunosenescence, reportedly caused by the overproduction of pro-inflammatory cytokines that are also responsible for the decrease in the formation of long-term memory B cells. The presence of elements that diminish the immune response, aside from those related to B-cell function, notably those appertaining to $\mathrm{T}$ cells, is particularly important for evaluating the long-term effectiveness of SARS-CoV-2 immunity, notably in the potentially immunosenescent population composed of institutionalized elderly patients.

It is vital to consider the cellular component of the immune response, which is predominant in viral respiratory infections (24). Indeed, T cells are essential in attenuating the severity of the disease, but may nonetheless have a role in severe manifestations if their activation is unregulated (25). The generation of memory $\mathrm{T}$ cells, including those against SARS$\mathrm{CoV}-2$, guarantees long-term immune protection, whereas the humoral response is susceptible to waning, as described for SARS and MERS-CoV (26). Even six months after SARSCoV-2 infection (27), specific CD4+ and CD8+ lymphocytes can be found in the vast majority of convalescent donors. Furthermore, a study that examined differences between the immune response of an immunocompetent patient and that of a patient suffering from common variable immunodeficiency (CVID), who both presented with a mild form of SARSCoV-2 infection, showed the absence of anti-SARS-CoV-2 IgG antibodies in the CVID patient, despite a normal absolute lymphocyte count. This study also reported a marked decrease in the proportion of germinal center B cells and plasma blasts in the CVID patient, offering a possible explanation for the absence of anti-SARS-CoV-2 antibodies (23). Accordingly, such an absence of antibodies in what are otherwise paucisymptomatic forms also supports the hypothesis that the T-cell response has the primary role in defending against SARSCoV-2 infection. Finally, a hypothesis has been formulated concerning the age-related sensitivity to SARS-CoV-2, proposing a diverse repertoire of naïve $\mathrm{T}$ cells to explain the protection from severe forms observed in children, while also explaining the higher risk in elderly patients, who are naturally affected by immunosenescence (28).

\section{Conclusion}

It is difficult to limit viral propagation during a SARSCoV-2 epidemic outbreak in geriatric care establishments. Elderly and dependent patients are at risk of developing severe disease due to their immune and clinical profile, making herd immunity in these institutions all the more essential. Nonetheless, persistent immunity via neutralizing antibodies may be possible in this population. Vaccination against SARS$\mathrm{CoV}-2$ induces an effective humoral response in cases in which the elderly patient has already developed anti SARS-CoV-2 antibodies, and suggests that a single vaccine dose may suffice. On the other hand, when this strategy is applied to a potentially immunosenescent seronegative patient, the antibody titer remains low, suggesting the need for supplementary doses. Future evaluation of vaccination and protective immunity in this population should include other markers of response aside from those related to $\mathrm{B}$ cells, in particular those related to $\mathrm{T}$ cells, including screening for a specific SARS-CoV-2 response. This would allow the consideration of novel vaccination strategies that associate humeral and cellular responses, thus enabling more persistent and effective immunity, while at the same time making the emergence of novel variants less likely.

Conflict of Interest: The authors declare no conflict of interest.

Source of funding: The study was funded by a donation from the AGIPI fund for COVID-19 solidarity. The AGIPI (association générale interprofessionnelle de prévoyance et d'investissement) is an organization created in 1976. Since then, it has acted to promote pensions, savings, providence, and health. AGIPI offers solutions that match individual and professional protection needs and has almost 620,000 members.

Compliance with Ethical Standards: This study was conducted in accordance with the guidelines of the local hospital ethics committee.

Authors' contributions: All authors made substantial contributions to the conception or design of the study or to the acquisition, analysis, or interpretation of data for the study; critically reviewed the data; and approved the final version to be published.

\section{References}

1. Mo P, Xing Y, Xiao Y, et al. (2020) Clinical characteristics of refractory COVID-19 pneumonia in Wuhan, China. Clin Infect Dis. doi:10.1093/cid/ciaa270

2. Morley JE, Vellas B. COVID-19 and Older Adult. J Nutr Health Aging 2020;24:364 365. https://doi.org/10.1007/s12603-020-1349-9

3. Etard J-F, Vanhems P, Atlani-Duault L, Ecochard R. Potential lethal outbreak of coronavirus disease (COVID-19) among the elderly in retirement homes and long-term facilities, France, March 2020. Euro Surveill. 2020;25(15). doi:10.2807/1560-7917. ES.2020.25.15.2000448

4. O'Driscoll M, Ribeiro Dos Santos G, Wang L, et al. Age-specific mortality and immunity patterns of SARS-CoV-2. Nature. 2021;590(7844):140-145. doi:10.1038/ s41586-020-2918-0

5. Plaçais L, Richier Q. [COVID-19: Clinical, biological and radiological characteristics in adults, infants and pregnant women. An up-to-date review at the heart of the pandemic]. Rev Med Interne. 2020;41(5):308-318. doi:10.1016/j.revmed.2020.04.004

6. Krammer F. SARS-CoV-2 vaccines in development. Nature. 2020;586(7830):516-527. doi:10.1038/s41586-020-2798-3

7. Baden LR, El Sahly HM, Essink B, et al. Efficacy and Safety of the mRNA1273 SARS-CoV-2 Vaccine. N Engl J Med. 2021;384(5):403-416. doi:10.1056/ NEJMoa2035389

8. Dan JM, Mateus J, Kato Y, et al. Immunological memory to SARS-CoV-2 assessed for up to 8 months after infection. Science. 2021;371(6529). doi:10.1126/science.abf4063

9. Polack FP, Thomas SJ, Kitchin N, et al. Safety and Efficacy of the BNT162b2 mRNA Covid-19 Vaccine. N Engl J Med. 2020;383(27):2603-2615. doi:10.1056/ NEJMoa2034577

10. Manisty C, Otter AD, Treibel TA, et al. Antibody response to first BNT162b2 dose in previously SARS-CoV-2-infected individuals. Lancet. 2021;397(10279):1057-1058. doi:10.1016/S0140-6736(21)00501-8

11. Saadat S, Rikhtegaran Tehrani Z, Logue J, et al. Binding and Neutralization Antibody Titers After a Single Vaccine Dose in Health Care Workers Previously Infected With SARS-CoV-2. JAMA. 2021;325(14):1467-1469. doi:10.1001/jama.2021.3341

12. Fülöp T, Dupuis G, Witkowski JM, Larbi A. The Role of Immunosenescence in the Development of Age-Related Diseases. Rev Invest Clin. 2016;68(2):84-91

13. Wikby A, Månsson IA, Johansson B, Strindhall J, Nilsson SE. The immune risk profile is associated with age and gender: findings from three Swedish population studies of individuals 20-100 years of age. Biogerontology. 2008;9(5):299-308. doi:10.1007/ s10522-008-9138-6

14. Wikby A, Nilsson B-O, Forsey R, et al. The immune risk phenotype is associated with IL-6 in the terminal decline stage: findings from the Swedish NONA immune longitudinal study of very late life functioning. Mech Ageing Dev. 2006;127(8):695704. doi: $10.1016 /$ j.mad.2006.04.003

15. Wikby A, Ferguson F, Forsey R, et al. An immune risk phenotype, cognitive impairment, and survival in very late life: impact of allostatic load in Swedish 
octogenarian and nonagenarian humans. J Gerontol A Biol Sci Med Sci. 2005;60(5):556-565. doi:10.1093/gerona/60.5.556

16. Rockwood K, Song X, MacKnight C, et al. A global clinical measure of fitness and frailty in elderly people. CMAJ. 2005;173(5):489-495. doi:10.1503/cmaj.050051

17. Brochot E, Demey B, Touzé A, et al. Anti-spike, Anti-nucleocapsid and Neutralizing Antibodies in SARS-CoV-2 Inpatients and Asymptomatic Individuals. Front Microbiol. 2020;11:584251. doi:10.3389/fmicb.2020.584251

18. Millet JK, Whittaker GR. Murine Leukemia Virus (MLV)-based Coronavirus Spikepseudotyped Particle Production and Infection. Bio Protoc. 2016;6(23). doi:10.21769/ BioProtoc. 2035

19. Varona JF, Madurga R, Peñalver F, et al. kinetics of anti-SARS-CoV-2 antibodies over time. Results of 10 month follow up in over 300 seropositive Health Care Workers. Eur J Intern Med. Published online May 25, 2021. doi:10.1016/j.ejim.2021.05.028

20. Matusali G, Colavita F, Lapa D, et al. SARS-CoV-2 Serum Neutralization Assay: A Traditional Tool for a Brand-New Virus. Viruses. 2021;13(4). doi:10.3390/v13040655

21. Dufloo J, Grzelak L, Staropoli I, et al. Asymptomatic and symptomatic SARSCoV-2 infections elicit polyfunctional antibodies. Cell Rep Med. 2021;2(5):100275. doi:10.1016/j.xcrm.2021.100275

22. Yamayoshi S, Yasuhara A, Ito M, et al. Antibody titers against SARS-CoV-2 decline, but do not disappear for several months. EClinicalMedicine. 2021;32:100734. doi:10.1016/j.eclinm.2021.100734
23. Gupta S, Su H, Narsai T, Agrawal S. SARS-CoV-2-Associated T-Cell Responses in the Presence of Humoral Immunodeficiency. Int Arch Allergy Immunol. 2021;182(3):195209. doi:10.1159/000514193

24. Schmidt ME, Varga SM. The CD8 T Cell Response to Respiratory Virus Infections. Front Immunol. 2018;9:678. doi:10.3389/fimmu.2018.00678

25. Laing AG, Lorenc A, Del Molino Del Barrio I, et al. A dynamic COVID-19 immune signature includes associations with poor prognosis. Nat Med. 2020;26(10):1623-1635 doi:10.1038/s41591-020-1038-6

26. Shin H-S, Kim Y, Kim G, et al. Immune Responses to Middle East Respiratory Syndrome Coronavirus During the Acute and Convalescent Phases of Human Infection. Clin Infect Dis. 2019;68(6):984-992. doi:10.1093/cid/ciy595

27. Zuo J, Dowell AC, Pearce H, et al. Robust SARS-CoV-2-specific T cell immunity is maintained at 6 months following primary infection. Nat Immunol. 2021;22(5):620626. doi:10.1038/s41590-021-00902-8

28. Vardhana SA, Wolchok JD. The many faces of the anti-COVID immune response. J Exp Med. 2020;217(6). doi:10.1084/jem.20200678

How to cite this article: J. Moyet, F. Helle, G. Bourdenet, et al. Kinetics of SARS-CoV2-Neutralising Antibodies of Residents of Long-Term Care Facilities. J Nutr Health Aging. 2022;26(1):57-63, http://dx.doi.org/10.1007/s12603-021-1713-4 